\title{
Inclusive diffraction and a measurement of the diffractive longitudinal structure function $F_{L}^{D}$ at HERA
}

\author{
Laurent Favart* \\ I.I.H.E., Université Libre de Bruxelles \\ 1050 Brussels \\ Belgium \\ E-mail: lfavart@ulb.ac.be
}

Results are reported on recent measurements of the cross section of the diffractive deep-inelastic process $e p \rightarrow e X p$ using different experimental methods. The longitudinal contribution to the diffractive cross section is shown for the first time.

European Physical Society Europhysics Conference on High Energy Physics, EPS-HEP 2009,

July 16 - 222009

Krakow, Poland

*On behalf of the H1 and ZEUS Collaborations. 


\section{Introduction}

The diffractive interaction is a feature of hadron-hadron scattering at high energy corresponding to a $t$-channel exchange of the vacuum quantum numbers and a small momentum transfer. It has been described, in the past, in the framework of Regge theory, where the exchange was interpreted as the Pomeron $(\mathbb{P})$ trajectory, characterized by a weak energy dependence, in particular, with respect to the fast decrease of the total cross section at smaller energy due to Reggeon $(\mathbb{R})$ exchange: $\sigma_{T o t}=B W^{2\left(\alpha_{\mathbb{R}}-1\right)}+A W^{2\left(\alpha_{\mathbb{P}}-1\right)}$, where $W$ is the center of mass energy, $A$ and $B$ normalisation factors and typically $\alpha_{\mathbb{R}}=0.55$ and $\alpha_{\mathbb{P}}=1.08$.

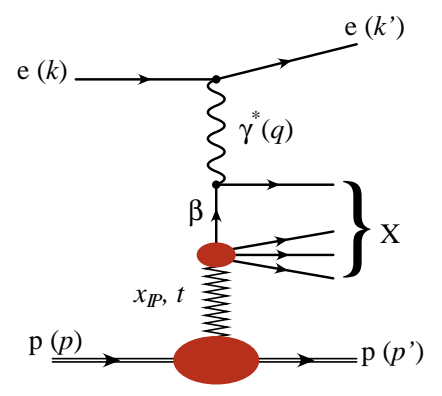

Figure 1: Basic diagrams for diffraction in presence of a hard scale at in $e p$ collisions.

Diffraction has two distinguishing features. First, hadron emission from the exchange itself being suppressed by its colourless nature, the two diffractively dissociated systems are separated in rapidity space, forming a large rapidity gap (LRG). Secondly, the diffractive events are observed with a small momentum transfer in both the transverse and longitudinal coordinates. The four-momentum of the exchange, $t$, and the longitudinal momentum fraction of the exchange, $x_{\mathbb{P}}$, are both small; $|t|$ is typically less than the square of the nucleon mass and $x_{\mathbb{P}}$ is smaller than 0.05 .

The high energies of the HERA ep collider allowed us for the first time to study diffraction in terms of perturbative QCD (pQCD), i.e. in the presence of a hard scale (called hard diffraction). At HERA the diffractive interaction takes place between the hadronic behaviour of the exchanged virtual photon and the proton (see Fig. 1).

This article concentrates on different measurements performed at HERA by the H1 and ZEUS Collaborations on inclusive hard diffraction. Their analysis and interpretation in terms of pQCD is discussed in [1].

\section{Kinematics and total cross section}

The inclusive diffractive cross section at HERA, $e p \rightarrow e X p$, can be defined with the help of four kinematic variables conveniently chosen as $Q^{2}, x_{\mathbb{P}}, \beta$ and $t$. The variable $\beta$ is the fraction of the exchanged momentum carried by the quark struck by the photon, or in other terms, the fraction of the exchanged momentum reaching the photon. These variables are related to the Bjorken $x_{B j}$ scaling variable by the relation $x_{B j}=\beta \cdot x_{\mathbb{P}}$. The presence of the hard scale, $Q^{2}$, ensures that the virtual photon is point-like and that the photon probes the partonic structure of the diffractive exchange (Fig. 1), in analogy with the inclusive DIS processes. Also by analogy, the measured cross section is expressed in the form of two four-fold diffractive structure functions $F_{2}^{D(4)}$ and $F_{L}^{D(4)}$ according to

$$
\frac{\mathrm{d}^{4} \sigma(e p \rightarrow e X p)}{\mathrm{d} Q^{2} \mathrm{~d} x_{\mathbb{P}} \mathrm{d} \beta \mathrm{d} t}=\frac{4 \pi \alpha^{2}}{\beta Q^{4}}\left[Y_{+} F_{2}^{D(4)}\left(Q^{2}, x_{\mathbb{P}}, \beta, t\right)-y^{2} F_{L}^{D(4)}\left(Q^{2}, x_{\mathbb{P}}, \beta, t\right)\right]
$$


where $Y_{+}=1+(1-y)^{2}$ and $y$ is the usual Bjorken variable, $y \simeq W^{2} / s$. The reduced cross section, used later, is defined as $\sigma_{r}^{D(4)}=F_{2}^{D(4)}-y^{2} / Y_{+} F_{L}^{D(4)}$. For $y$ not too close to unity, $\sigma_{r}^{D(4)}=F_{2}^{D(4)}$ holds to very good approximation.

\section{Cross section measurements and comparisons}

Experimentally, diffractive processes are accessed either by tagging the outgoing proton in the H1 Forward Proton Spectrometer or the ZEUS Leading Proton Spectrometer ('LPS method' $[2,4,5])$ or by requiring the presence of a large gap in the rapidity distribution of hadronic final state particles in the forward region ('LRG method' $[3,4,6])$. In a third approach, not considered in detail here, is based on the $M_{X}$ distribution shape [6, 7].
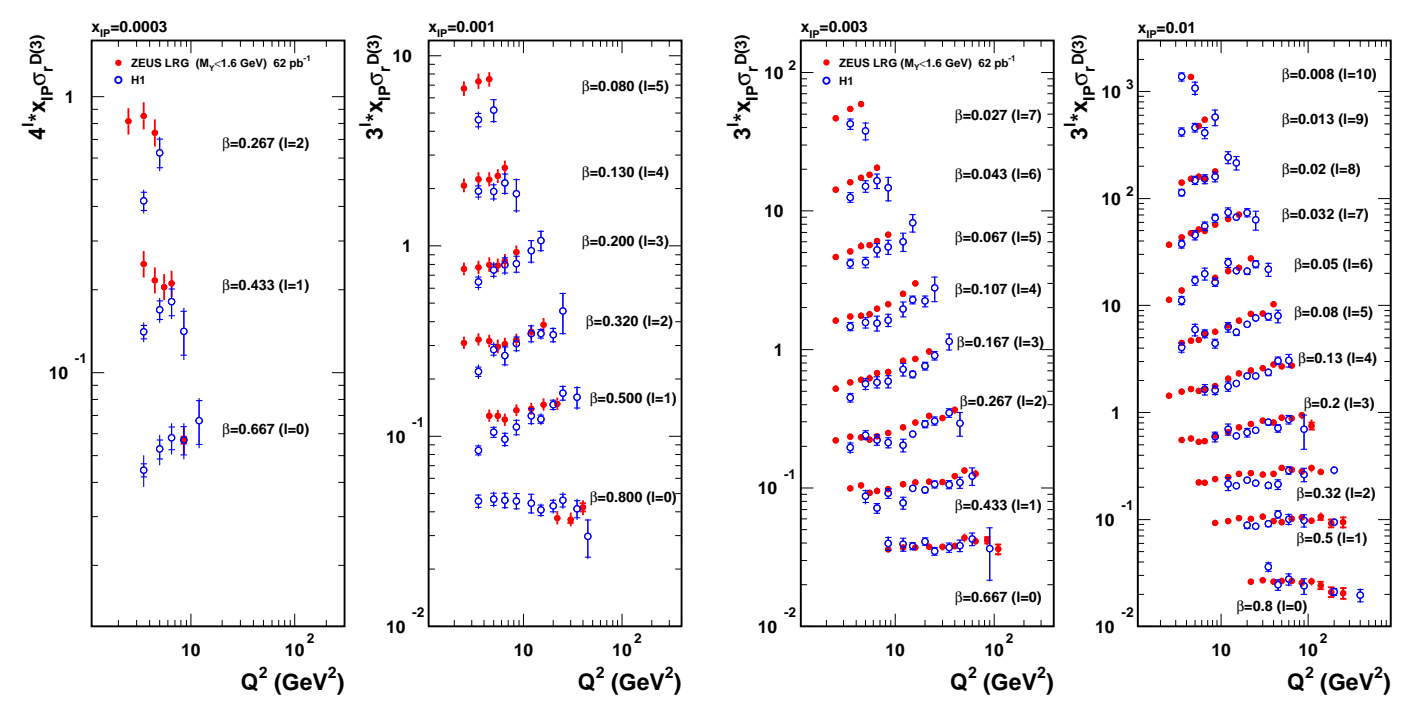

Figure 2: H1[3] and ZEUS[4] measurements of the diffractive reduced cross section.

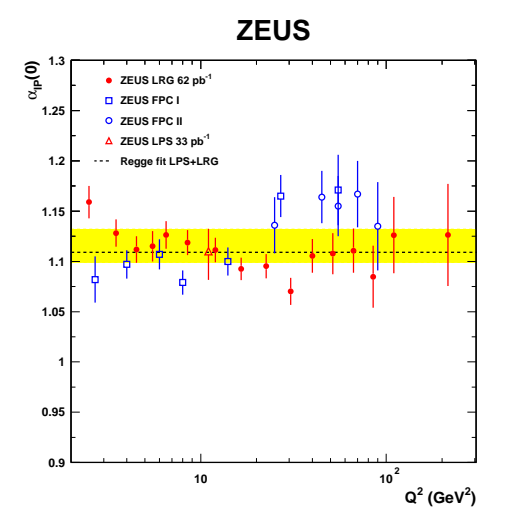

Figure 3: Pomeron intercept for different $Q^{2}$ values.
Whilst the LRG and $M_{X}$-based techniques yield better statistics than the LPS method, they suffer from systematic uncertainties associated with an admixture of proton dissociation to low mass states, which is irreducible due to the limited forward detector acceptance.

The H1 collaboration recently released a preliminary proton-tagged measurement using its full available FPS sample at HERA-II [5]. The integrated luminosity is $156 \mathrm{pb}^{-1}$, a factor of 20 beyond previous $\mathrm{H} 1$ measurements. The new data tend to lie slightly above the recently published final ZEUS LPS data from HERA-I [4], but are within the combined normalisation uncertainty of around $10 \%$. The $t$ dependence of the cross section is not correlated to the other kinematic variables (i.e. $Q^{2}, x_{\mathbb{P}}$ or $\beta$ ) $[2,4]$ allowing the factorisation $\sigma_{r}^{D(4)}=\sigma_{r}^{D(3)} e^{-b|t|}$. 
Using the LRG method, the recently published ZEUS measurements [4] are based on an integrated luminosity of $62 \mathrm{pb}^{-1}$ and thus have substantially improved statistical precision compared with the older H1 published results [3]. The normalisation differences between the two experiments are most obvious here, having been quantified at 13\%, which is a little beyond one standard deviation in the combined normalisation uncertainty. After correcting for this factor, very good agreement is observed between the shapes of the H1 and ZEUS cross sections throughout most of the phase space studied, as shown in figure 2. A DGLAP QCD fit is applied to these measured values to extract the diffractive parton densities (DPDFs) as discussed in the contribution of A. Garfagnini to this conference [1].

Performing a fit of the cross section with a form à la Regge (see introduction) one founds that the Pomeron intercept, $\alpha_{I P}$ does not depend on $Q^{2}$ (see Fig.3 and [4]), in strong contrast to what is measured in inclusive DIS. This confirms the dominance of non-perturbative effects in the Pomeron.

\section{The longitudinal diffractive structure function}

At low $x$ and $Q^{2}$, the longitudinal diffractive structure function, $F_{L}^{D}$, is closely related to the diffractive gluon density and thus gives a complementary test of diffractive factorisation and the role of gluons to those provided by jet and charm cross sections. Measurements of $F_{L}^{D}$ became possible following the reduced proton beam energy runs at the end of HERA operation.

The H1 collaboration recently released preliminary $F_{L}^{D}$ data, as shown in figure 4 . The results [8], when integrated over $\beta$ show that $F_{L}^{D}$ is non-zero at the $3 \sigma$ level. It is also clearly incompatible with its maximum possible value of $F_{2}^{D}$.

\section{References}

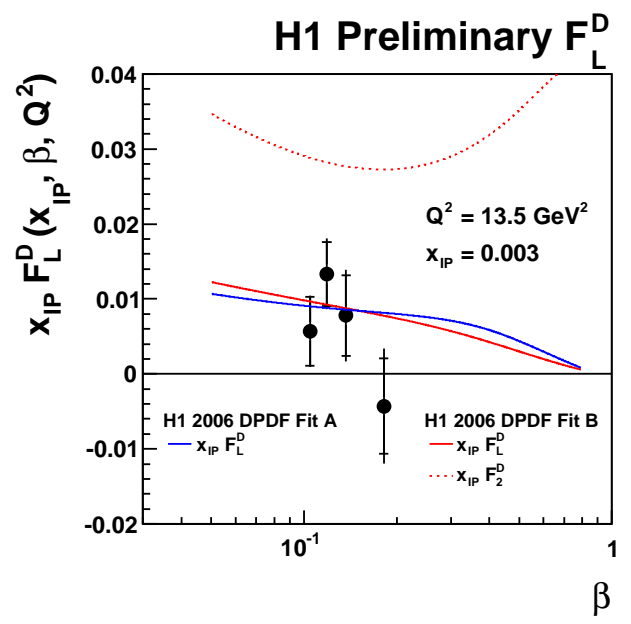

Figure 4: First $F_{L}^{D}$ measurement [8], compared with DPDF based predictions.

[1] A. Garfagnini, these proceedings.

[2] H1 Coll., Eur. Phys. J. C 48 (2006) 749 [hep-ex/0606003].

[3] H1 Coll., Eur. Phys. J. C 48 (2006) 715 [hep-ex/0606004].

[4] ZEUS Coll., Nucl. Phys. B 816 (2009) 1 [hep-ex/0812.2003].

[5] H1 Coll., 'Measurement of diffractive DIS with a leading proton at HERA-2' [H1prelim-09-012].

[6] H1 Coll., 'Measurement of Inclusive Diffractive DIS at HERA (99-04 data)' [H1prelim-06-014].

[7] ZEUS Coll., Nucl. Phys. B 800 (2008) 1 [hep-ex/0802.3017].

[8] H1 Coll., 'Measurement of $F_{L}^{D}$ at HERA II' [H1 prelim-09-011]. 